\title{
Reduced cortical thickness of the paracentral lobule in at-risk mental state individuals with poor 1-year functional outcomes
}

Daiki Sasabayashi iD ${ }^{1,2}$, Yoichiro Takayanagi (D) ${ }^{1,3}$, Tsutomu Takahashi (iD ${ }^{1,2}$, Shimako Nishiyama ${ }^{1,4}$, Yuko Mizukami ${ }^{1}$, Naoyuki Katagiri ${ }^{5}$,

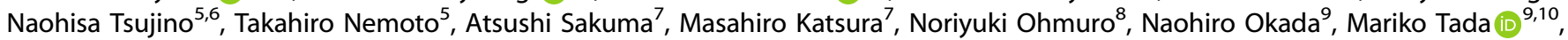
Motomu Suga ${ }^{9,11}$, Norihide Maikusa (D) $^{12}$, Shinsuke Koike (DD ${ }^{9,12}$, Atsushi Furuichi ${ }^{1,2}$, Mikio Kido ${ }^{1,2}$, Kyo Noguchi ${ }^{13}$, Hidenori Yamasue (iD ${ }^{9,14}$, Kazunori Matsumoto ${ }^{15}$, Masafumi Mizuno ${ }^{5}$, Kiyoto Kasai ${ }^{9,10}$ and Michio Suzuki ${ }^{1,2}$

(c) The Author(s) 2021

Although widespread cortical thinning centered on the fronto-temporal regions in schizophrenia has been reported, the findings in at-risk mental state (ARMS) patients have been inconsistent. In addition, it remains unclear whether abnormalities of cortical thickness (CT) in ARMS individuals, if present, are related to their functional decline irrespective of future psychosis onset. In this multicenter study in Japan, T1-weighted magnetic resonance imaging was performed at baseline in 107 individuals with ARMS, who were subdivided into resilient (77, good functional outcome) and non-resilient (13, poor functional outcome) groups based on the change in Global Assessment of Functioning scores during 1-year follow-up, and 104 age- and sex-matched healthy controls recruited at four scanning sites. We measured the CT of the entire cortex and performed group comparisons using FreeSurfer software. The relationship between the CT and cognitive functioning was examined in an ARMS subsample $(n=70)$. ARMS individuals as a whole relative to healthy controls exhibited a significantly reduced $\mathrm{CT}$, predominantly in the fronto-temporal regions, which was partly associated with cognitive impairments, and an increased CT in the left parietal and right occipital regions. Compared with resilient ARMS individuals, non-resilient ARMS individuals exhibited a significantly reduced CT of the right paracentral lobule. These findings suggest that ARMS individuals partly share CT abnormalities with patients with overt schizophrenia, potentially representing general vulnerability to psychopathology, and also support the role of cortical thinning in the paracentral lobule as a predictive biomarker for short-term functional decline in the ARMS population.

Translational Psychiatry (2021)11:396; https://doi.org/10.1038/s41398-021-01516-2

\section{INTRODUCTION}

Current evidence supports that early detection and intervention can lead to less severe negative symptomatology and better functional outcomes in patients with schizophrenia [1, 2], potentially by preventing and/or ameliorating active brain changes at early stages of psychosis [3-5]. However, brain morphological characteristics possibly associated with early neurodevelopmental abnormalities, such as altered sulco-gyral patterns [6-9], may also be related to a lower rate of remission and poor outcomes in schizophrenia. Recent high-risk studies further demonstrated that the majority of individuals with at-risk mental state (ARMS) [10,11] do not develop psychosis [12] but experience long-term functional impairments [13] due to several factors such as persistent subthreshold symptoms [14], comorbid depression and anxiety [15], and socio-cognitive dysfunction [16]. Whereas the onset of psychosis has been the outcome measure of interest in almost all previous high-risk studies [17], functional impairment has also been gaining attention as another important indicator [18], as it is associated with the development of persistent and recurrent nonpsychotic disorders that are commonly seen in ARMS and require ongoing clinical care [19]. However, neural underpinnings associated with functional outcomes in ARMS individuals remain largely unknown.

Cortical thickness (CT) may be a more sensitive measure of subtle cortical changes than gray matter volume [20, 21]. Schizophrenia patients likely exhibit widespread cortical thinning centered on the fronto-temporal regions [22], where baseline findings at first-episode $[23,24]$ and/or excessive thinning during the course of illness [25] may partly underlie cognitive impairments [26-28] and poor clinical outcomes [29, 30]. However, it remains unclear whether such $\mathrm{CT}$ abnormalities precede the onset of psychosis due to inconsistent findings [31-33] and whether CT

\footnotetext{
${ }^{1}$ Department of Neuropsychiatry, University of Toyama Graduate School of Medicine and Pharmaceutical Sciences, Toyama, Japan. ${ }^{2}$ Research Center for Idling Brain Science, University of Toyama, Toyama, Japan. ${ }^{3}$ Arisawabashi Hospital, Toyama, Japan. ${ }^{4}$ Center for Health Care and Human Sciences, University of Toyama, Toyama, Japan. ${ }^{5}$ Department of Neuropsychiatry, Toho University School of Medicine, Tokyo, Japan. ${ }^{6}$ Department of Psychiatry, Saiseikai Yokohamashi Tobu Hospital, Kanagawa, Japan. ${ }^{7}$ Department of Psychiatry, Tohoku University Hospital, Sendai, Japan. ${ }^{8}$ Osaki Citizen Hospital, Miyagi, Japan. ${ }^{9}$ Department of Neuropsychiatry, Graduate School of Medicine, The University of Tokyo, Tokyo, Japan. ${ }^{10}$ International Research Center for Neurointelligence (WRI-IRCN), UTIAS, The University of Tokyo, Tokyo, Japan. ${ }^{11}$ Graduate School of Clinical Psychology, Teikyo Heisei University, Tokyo, Japan. ${ }^{12}$ Center for Evolutionary Cognitive Sciences (ECS), Graduate School of Art and Sciences, The University of Tokyo, Tokyo, Japan. ${ }^{13}$ Department of Radiology, University of Toyama Graduate School of Medicine and Pharmaceutical Sciences, Toyama, Japan. ${ }^{14}$ Department of Psychiatry, Hamamatsu University School of Medicine, Hamamatsu, Japan. ${ }^{15}$ Kokoro no Clinic OASIS, Sendai, Japan. ${ }^{\circledR}$ email: ds179@med.u-toyama.ac.jp
} 
findings in ARMS individuals are related to cognitive function and/ or functional outcomes. A recent study by de Wit et al. [34] reported the association between widespread cortical thinning at baseline and poor functional outcomes at 6-year follow-up in a relatively small ARMS cohort $(n=35)$, but a potential relationship between the CT abnormalities in ARMS individuals, if present, and their shorter-term outcomes remains largely unknown.

In the present study, we investigated CT abnormalities at baseline and their relevance to functional outcomes at the 1-year follow-up in a relatively large sample of ARMS individuals recruited at four Japanese scanning sites. We also examined the relationship between the $C T$ findings and cognitive functioning in an ARMS subsample. Based on the previous findings in ARMS individuals [31, 34], we predicted widespread CT reduction, especially in ARMS individuals with poor functional outcomes. Based on previous findings in schizophrenia [27, 28], we also predicted that cortical thinning centered on the frontal region is related to specific cognitive impairments in the ARMS population.

\section{MATERIALS AND METHODS Participants}

From September 2006 to August 2012, we recruited 107 ARMS individuals at four hospitals with specialized ARMS services [35, 36]: Toyama University Hospital, Toho University Hospital, Tohoku University Hospital, and The University of Tokyo Hospital (Table 1) [37]. A consensus diagnosis of ARMS for all patients was provided by clinical research meetings at each site based on the interview data using the Comprehensive Assessment of AtRisk Mental States (CAARMS) [10] (Toyama and Tohoku) or the Structured Interview for Prodromal Symptoms/the Scale of Prodromal Symptoms [38] (Toho and Tokyo). ARMS individuals were evaluated using the Global Assessment of Functioning (GAF) [39] both at MRI (baseline) and a year after (follow-up), and classified into good (ARMS-resilient [ARMS-R], baseline score $\leq$ follow-up score, $n=77$ ) or poor (ARMS-non-resilient [ARMS-NR], baseline score $>$ follow-up score, $n=13$ ) functional outcome subgroups. During the follow-up period (mean $=4.9$ years, $S D=2.6$ years), 21 ARMS individuals (14 ARMS-R and 3 ARMS-NR individuals) subsequently developed psychosis, whose diagnoses using the Diagnostic and Statistical Manual of Mental Disorders-Fourth Edition (DSM-IV) [40] were schizophrenia $(n=14)$, delusional disorder $(n=1)$, schizophreniform disorder $(n$ $=1)$, brief psychotic disorder $(n=1)$, and psychotic disorder not otherwise specified $(n=4)$. At baseline, a substantial number of ARMS individuals ( $n$ $=43 / 107,40.2 \%$ ) were using the minimum dosage of antipsychotics for their mental condition (e.g., rapid deterioration or suicidal and harmful risk) according to previous reports [41]. Cognitive function was rated at baseline using Japanese version of the Brief Assessment of Cognition in Schizophrenia (BACS) [42] in a subsample of ARMS individuals $(n=70)$. We used raw scores for the six BACS subdomains: (i) list learning as verbal memory, (ii) digit sequencing task as working memory, (iii) token motor task as motor speed, (iv) category and letter fluency as verbal fluency, (v) symbol coding as attention and processing speed, and (vi) the Tower of London task as executive function. We also recruited 104 age- and sex-matched healthy controls from the community, hospital staff, and university students at each site (Table 1). All participants were physically healthy, and none had a history of serious head injury, neurological illness, substance abuse or dependence (including cannabis use) disorder, or previous psychotic episode that fulfilled the DSM-IV criteria. All subjects were included in our previous study that assessed the subcortical volumes in ARMS individuals [43]. After a complete explanation of the design and purpose of the study, which was approved by the medical ethics committee of each site, written informed consent was received from all participants.

\section{Image acquisition and processing}

The subjects underwent 1.5- (Toyama, Toho, and Tohoku) and 3-Tesla (Tokyo) MRI using the protocols at each site (Supplementary Table 1). Obtained T1-weighted MR scans were preprocessed by FreeSurfer software (ver.5.3.) [44]. The implemented processing stream was involved in removal of non-brain tissue, transformation to Talairach-like space, segmentation of gray and white matter tissue, triangular tessellateion, and inspection of the white matter and pial surfaces. One trained researcher (DS) who was blinded to the subjects' identities visually inspected the reconstructed images and manually corrected any errors of the cortical segmentations. Each vertex-wise CT value, which was computed as the minimum distance between pial and white surfaces for each vertex, was mapped on a common spherical coordinate system smoothed with a 10-mm full width at half-maximum Gaussian kernel.

\section{Statistical analysis}

Statistical testing for demographic differences among groups was conducted by one-way ANOVA or chi-square tests.

Potential contribution of baseline clinical variables (positive symptoms subscale scores of the CAARMS $[n=50]$ or SOPS $[n=39$ ] and BACS subscores $[n=63]$ ) to functional outcomes (i.e., follow-up GAF score at 1 year) was tested using univariate linear regression analyses in SPSS (ver. 22.0.0) (IBM Corp., Armonk, NY). The significance threshold was set at $p<$ 0.05 (two-tailed) and a Bonferroni correction was applied to prevent a possible type I error due to multiple tests.

Within FreeSurfer's Query Design Estimate Contrast application, a general linear model controlling for age, sex, and scanning sites was used to estimate group differences in the CT value at each vertex. Vertex-byvertex whole-brain $C T$ correlation analyses with baseline clinical variables (duration of education [ $n=209]$, antipsychotic medication dose [ $n=43]$, positive symptoms subscale scores of the CAARMS $[n=55]$ or SOPS $[n=$ 50], and BACS subscores $[n=70]$ ) were also carried out by a general linear model controlling for age, sex, and scanning sites. A Monte Carlo Simulation, which was embedded in the Analysis of Functional Neurolmages' AlphaSim program (NIMH, Bethesda, MD, USA), was applied to correct for multiple comparisons [45]. To define significant clusters, 10,000 iterations of simulations were employed for each comparison whose significance threshold was set at $p<0.05$ (two-tailed and corrected for multiple testing).

In order to overcome the problem of inter-site bias, we conducted two corroborative analyses. First, we adapted a prospective meta-analysis approach similar to recent multisite studies [46, 47] using SPSS (ver. 22.0.0) and Review Manager (ver. 5.3) (the Nordic Cochrane Centre, Cochrane Collaboration, Copenhagen, Denmark). Each CT value was extracted from ten regions of interest (ROls), where significant group differences in $\mathrm{CT}$ were detected (Table 2). Group differences in CT in the ROls were investigated by each site and Cohen's $d$ effect sizes were calculated from the group contrasts. Those for each site were entered into a random-effect model meta-analysis and overall effect sizes were obtained. Second, we employed a Combat algorithm, which was a novel and reliable harmonization technique for combining brain measures across multisite $[48,49]$, to remove unwanted scanner variations. The harmonized CT values in the ten ROIs (Table 2) were compared across the groups using ANCOVA with age and sex as covariates. We also performed an unsupervised dimension reduction of the $\mathrm{CT}$ measurement using principal component analysis to visualize how the variation in the data was related to scanning site before and after the harmonization.

Given the previously reported findings of larger cortical surface area (SA) for ARMS-R compared to ARMS-NR [34], we also conducted group comparisons in the SA values using a similar general linear model.

\section{RESULTS}

\section{Demographic background}

Groups were matched for age, sex, and parental education, but the healthy controls were characterized by higher education and higher rate of dextrality than the whole ARMS group (Table 1). The ARMS-R and -NR groups did not differ in baseline GAF score, medication status, rate of developing psychosis, $X^{2}(1)=0.17, p=$ 0.68 , or BACS subscores, but the ARMS-NR individuals exhibited fewer severe unusual thoughts at baseline and lower GAF score at follow-up than the ARMS-R individuals (Table 1).

\section{Baseline clinical variables and outcome}

Univariate linear regression analyses of ARMS subsamples demonstrated no significant contribution of baseline CAARMS ( $n$ $=50)$, SOPS $(n=39)$, or BACS $(n=63)$ subscores to follow-up GAF scores (Supplementary Table 2).

\section{Group comparison of CT}

Compared with the controls, the ARMS group as a whole exhibited a significantly reduced $C T$ in the frontal pole, superior frontal gyri, 


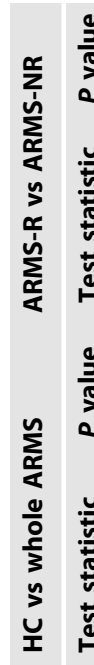

舫

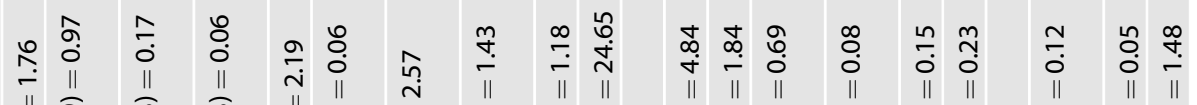

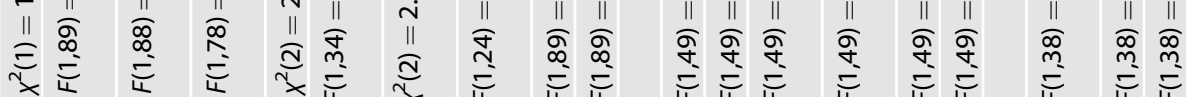

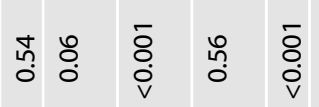

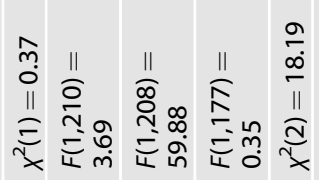

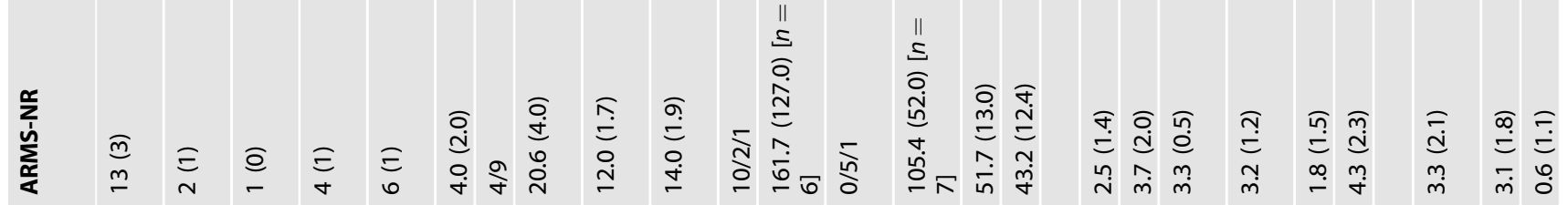

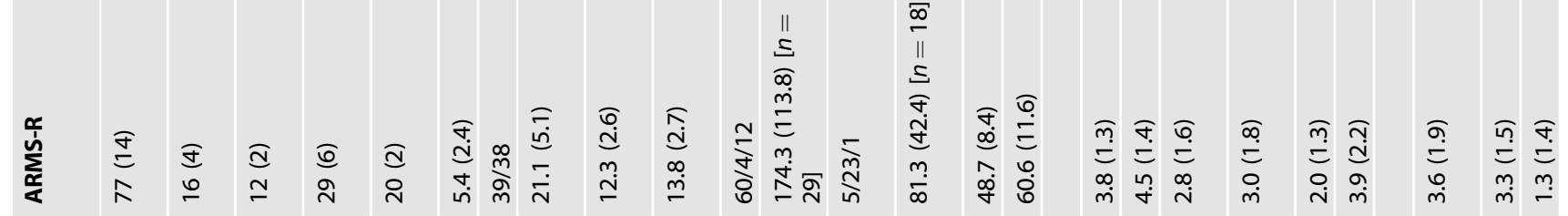

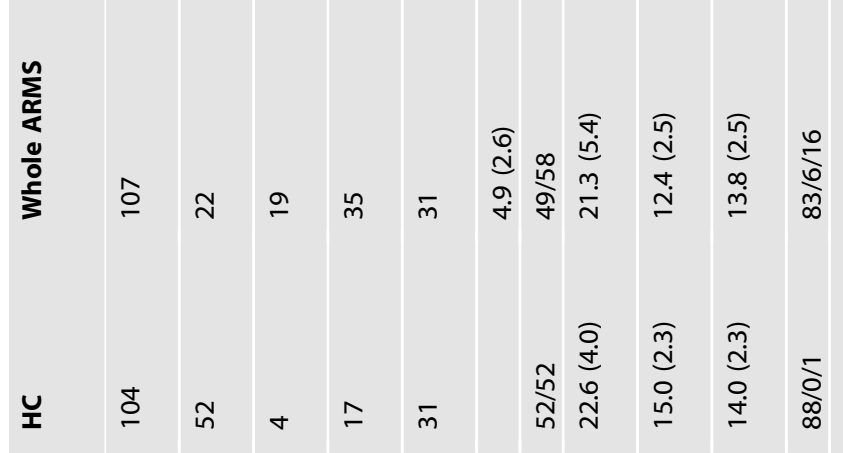
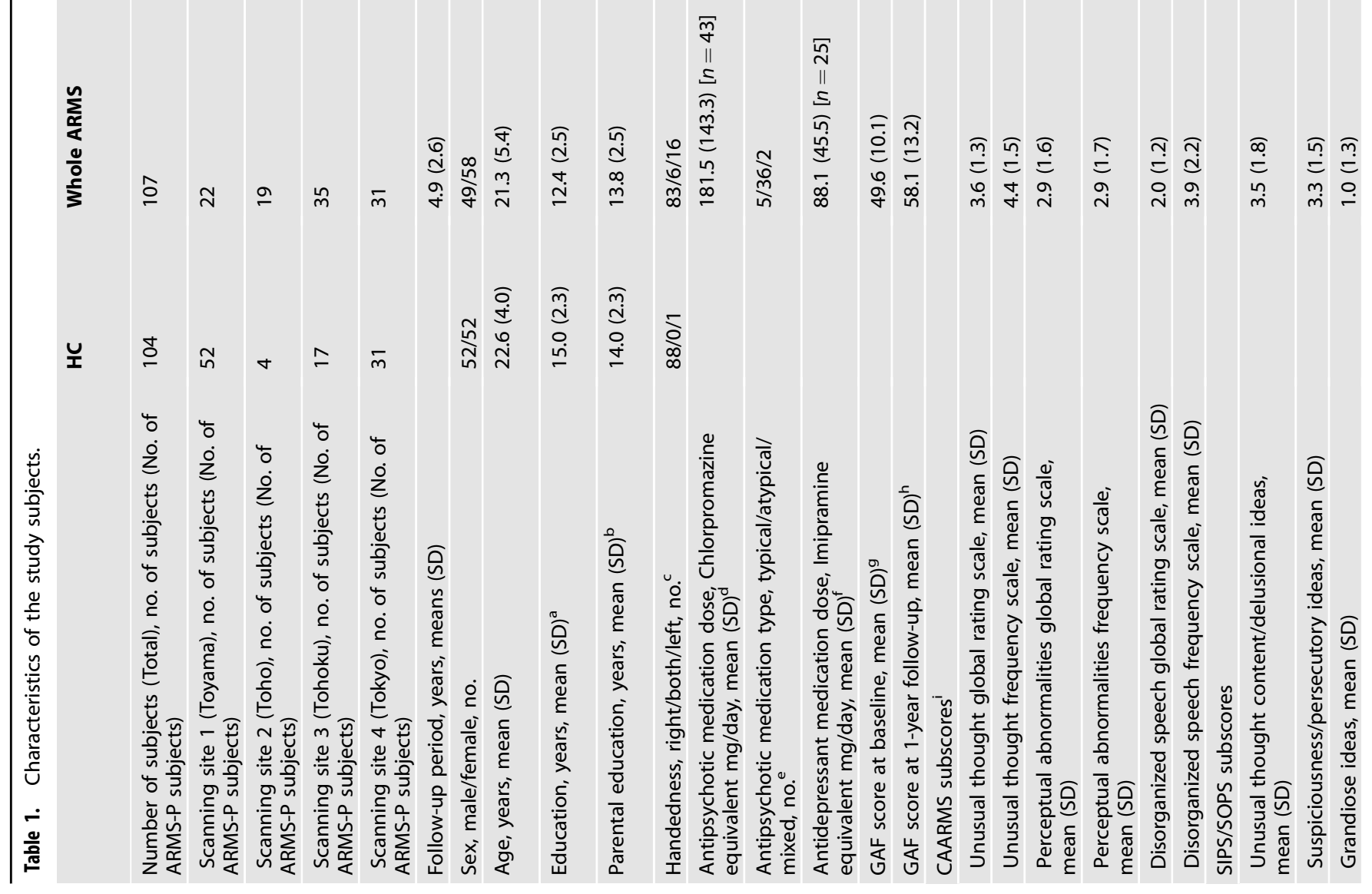


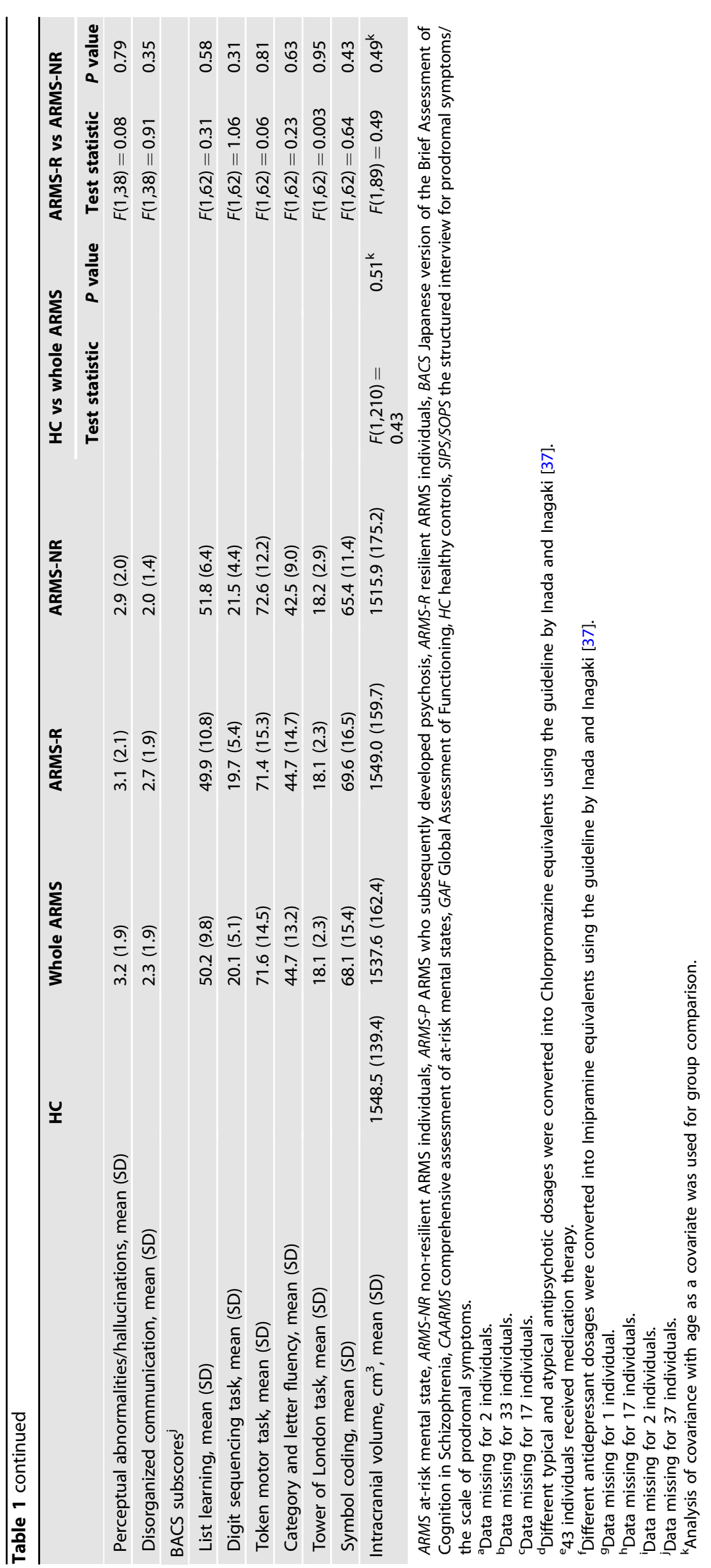


Table 2. Clusters with significant group differences in cortical thickness.

\begin{tabular}{|c|c|c|c|c|c|c|}
\hline \multirow[t]{2}{*}{ Cluster no. } & \multirow[t]{2}{*}{ Location of peak vertex (cluster name) } & \multicolumn{3}{|c|}{ MNI coordinates } & \multirow[t]{2}{*}{ Cluster size $\left(\mathrm{mm}^{2}\right)$} & \multirow{2}{*}{$\begin{array}{l}\text { Cluster-wise probability } \\
P \text { value }\end{array}$} \\
\hline & & $x$ & $y$ & $z$ & & \\
\hline \multicolumn{7}{|l|}{ ARMS $<\mathrm{HC}$} \\
\hline No. 2 & Left frontal pole & -8.6 & 61.3 & -9.2 & 4448.44 & 0.0001 \\
\hline No. 3 & Left insula & -27.7 & 22.9 & 4.3 & 1243.00 & 0.0028 \\
\hline No. 4 & Right fusiform & 36.7 & -39.6 & -10.7 & 4906.34 & 0.0001 \\
\hline No. 6 & Right precuneus & 10.2 & -46.9 & 28.8 & 869.66 & 0.0419 \\
\hline \multicolumn{7}{|l|}{ ARMS $>\mathrm{HC}$} \\
\hline No. 7 & Left postcentral & -19.9 & -28.8 & 58.5 & 1118.75 & 0.0056 \\
\hline No. 8 & Left precentral & -42.0 & -9.0 & 31.6 & 932.70 & 0.0198 \\
\hline No. 9 & Right pericalcarine & 17.9 & -71.3 & 11.3 & 1097.77 & 0.0088 \\
\hline
\end{tabular}

ARMS at-risk mental state, ARMS-NR non-resilient ARMS individuals, ARMS-R resilient ARMS individuals, HC healthy controls, MNI Montreal Neurological Institute.

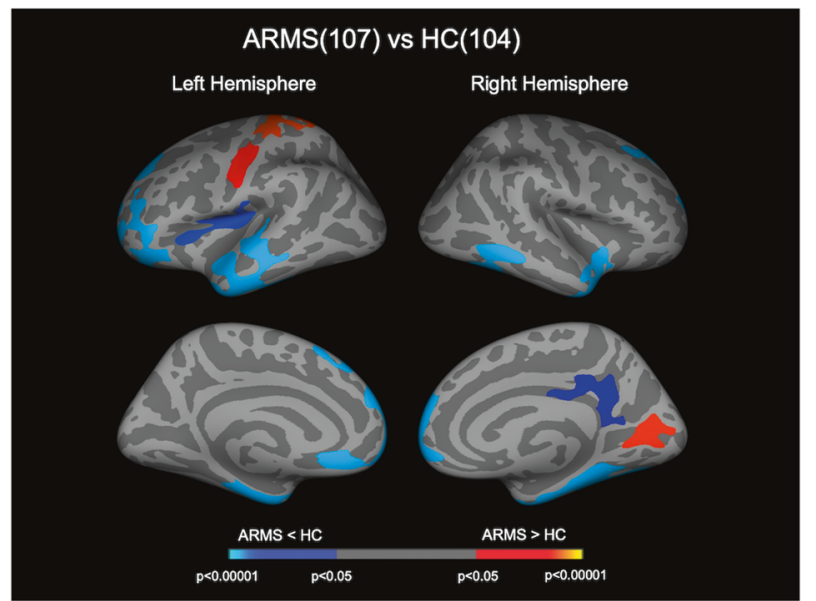

Fig. 1 Clusters showing differences in CT between ARMS individuals and HC. Cortical statistical maps displaying altered CT in individuals with ARMS compared with HC. The maps are shown for the right and left hemispheres in lateral and medial views, respectively. The horizontal bar shows $p$ values corrected for multiple comparisons. ARMS at-risk mental state, CT cortical thickness, $\mathrm{HC}$ healthy control.

orbitofrontal cortices, entorhinal cortices, parahippocampal gyri, fusiform gyri, lateral temporal gyri, temporal pole, and insular cortices bilaterally, and in the left middle frontal gyrus, right posterior and isthmus cingulate gyri, and right precuneus cortex. They also exhibited a significantly increased CT in the left pre- and postcentral gyri, right lingual gyrus, and right pericalcarine cortex (Fig. 1 and Table 2).

There were no significant differences in CT between the ARMS individuals who later developed psychosis (ARMS-P) and those who did not (ARMS-NP) at baseline.

The ARMS-NR group exhibited a significantly reduced $C T$ in the right paracentral lobule compared with the ARMS-R group (Fig. 2 and Table 2). This cluster remained significant even when we strictly defined the ARMS-NR (baseline GAF score $>$ follow-up score and follow-up score $<65, n=12$ ) and ARMS-R (baseline score $\leq$ follow-up score and follow-up score $\geq 65, n=31$ ) subgroups according to previous reports [30] (Supplementary Fig. 1). As supplementary analyses, we performed group comparisons

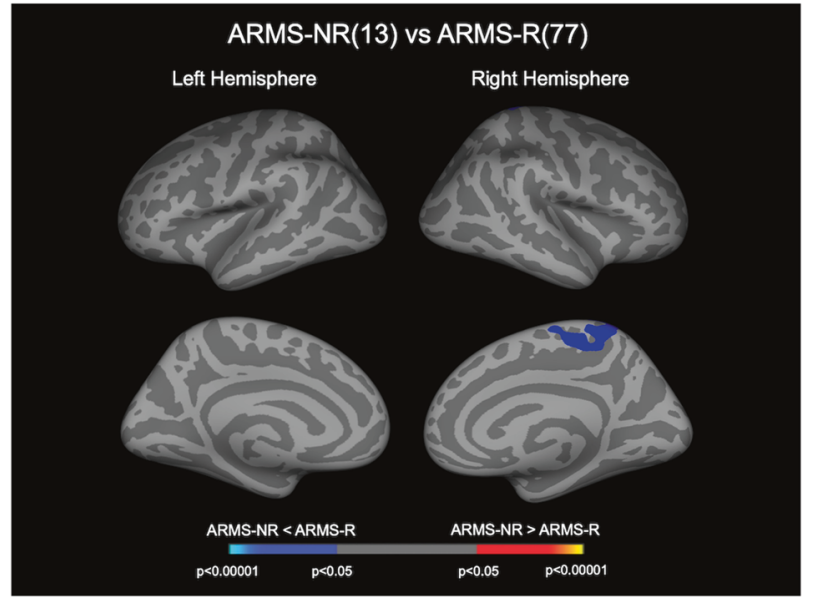

Fig. 2 Cluster showing difference in CT between ARMS-R and ARMS-NR. Cortical statistical maps displaying reduced CT in the ARMS-NR group compared with the ARMS-R group. The horizontal bar shows $p$ values corrected for multiple comparisons. ARMS at-risk mental state, ARMS-NR non-resilient ARMS individuals, ARMS-R resilient ARMS individuals, $C T$ cortical thickness.

(e.g., ARMS-R vs. controls, ARMS-NR vs. controls, and ARMS-P vs. $A R M S-N P$ ) in the mean $C T$ values of the cluster in the right paracentral lobule as well as correlation analyses between those and behavioral or clinical variables (duration of education, antipsychotic medication dosage, CAARMS or SOPS, and BACS subscores); although there were no significant differences between ARMS-R and controls or between ARMS-P and ARMSNP, ARMS-NR exhibited significantly lower CT values compared to controls (Supplementary Tables 3-5). No significant relationships were found between the CT value in this extracted cluster and behavioral or clinical variables. There were no regions where the ARMS-NR group exhibited a significantly increased CT compared with the ARMS-R group.

Supplementary analyses using meta-analytic overall effect sizes and Combat harmonization demonstrated no major effects of scanning site on the CT findings in this study (Supplementary Tables 6, 7 and Supplementary Figs. 2, 3). There was no obvious clustering by scanning site even in the raw data points (with a few 


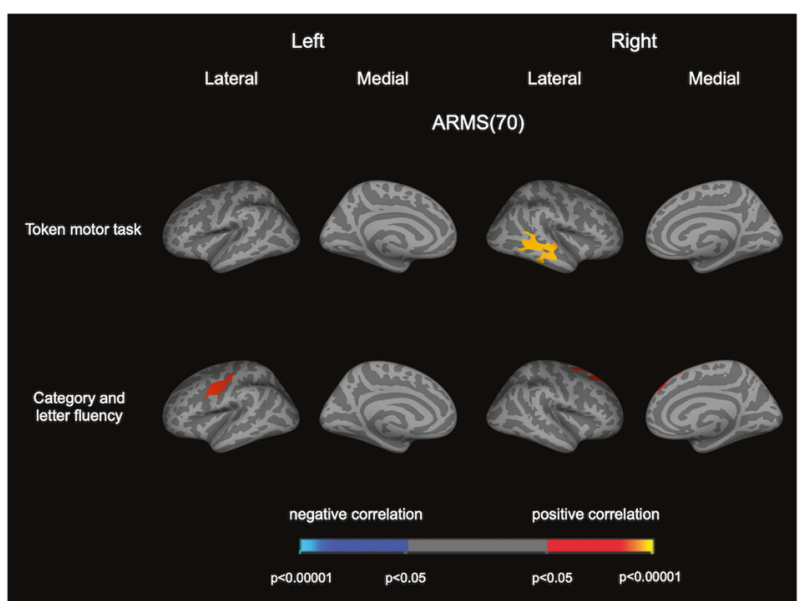

Fig. 3 Cortical statistical maps displaying the relationship between CT and cognitive performance in the ARMS group. Cortical statistical maps displaying the relationship between CT and BACS subscores (token motor task and verbal fluency) in individuals with ARMS. The horizontal bar shows $p$ values corrected for multiple comparisons. ARMS at-risk mental state, BACS Japanese version of the Brief Assessment of Cognition in Schizophrenia, CT cortical thickness.

exceptions in Toho University), and the harmonized data points were equally distributed across sites (Supplementary Fig. 4).

\section{Relationship of CT with cognitive measures and clinical variables}

For the BACS subscores, the Token motor task scores were positively correlated with $\mathrm{CT}$ in the right superior and middle temporal gyrus, and the Category and letter fluency scores were positively correlated with $\mathrm{CT}$ in the left precentral gyrus and right superior frontal gyrus in the entire ARMS population (Fig. 3). The other BACS subscores, antipsychotic medication dosage, CAARMS or SOPS subscores, or duration of education were not associated with $\mathrm{CT}$ in any region.

\section{Group comparison of SA}

There were no significant differences in SA between the ARMS and controls or between ARMS-R and ARMS-NR.

\section{DISCUSSION}

This is, to the best of our knowledge, the first multicenter MRI study to investigate the association between CT abnormalities and subsequent functional outcomes in a relatively large clinical highrisk cohort. ARMS individuals as a whole had a significantly reduced $\mathrm{CT}$, mainly in the fronto-temporal areas, and an increased CT in the parieto-occipital areas, which were partially associated with cognitive functioning. The ARMS-NR group had a significantly reduced $C T$ of the right paracentral lobule compared with the ARMS-R group, which may be a predictor of the short-term functional outcome irrespective of the manifestation of overt psychotic symptoms.

Reduced fronto-temporal CT in our ARMS cohort, which was similar to CT findings in schizophrenia [22], was consistent with previous high-risk studies [31, 32, 50], albeit with negative findings in other ARMS cohorts [33,51]. Reduced CT may be prominent, particularly in the ARMS-P group [52-55], but the present and previous studies $[32,51,56]$ found no $C T$ difference between the ARMS-P and -NP groups at baseline, although one of those studies showed progressive regional cortical thinning in the ARMS-P group [51]. These discrepancies may be partly due to the heterogeneity of the ARMS cohorts; our cohort was characterized by severe subthreshold symptomatology relative to those with negative $C T$ findings $[33,51]$. It should also be noted that fewer ARMS-P subjects ( $n=3 / 21$ [14.3\%]) received antidepressants, which can induce cortical thinning [57], than ARMS-NP subjects $(n=20 / 72$ [27.8\%]). Consistent with several studies on schizophrenia and ARMS $[58,59]$, we also noted increased CT in the left parietal and right occipital regions in the ARMS group relative to the controls. Parietal and occipital cortices may be comparatively preserved from progressive pathological processes $[60,61]$ because cortical maturation of these regions is mostly completed by the time of psychosis onset [62]. Taken together, our study supports ARMS subjects sharing bidirectional alterations of CT with patients with overt schizophrenia, which may reflect a complicated neurodevelopmental misbalance and its compensatory change across different brain areas [59].

The most important finding in this study was that a reduced CT of the right paracentral lobule distinguished the ARMS-NR subgroup from the ARMS-R subgroup. Although there were methodological differences (e.g., definition of "resilience", surfacebased ROI analysis vs. vertex-wise analysis), the present study replicated the findings by de Wit et al. [34] in demonstrating a reduced $C T$ specific to the non-resilient ARMS group, which may be due to disturbed compensatory neural mechanisms [34]. Although they found broad CT differences in the frontal and temporo-parietal regions between resilient and non-resilient individuals, our study suggested a specific role of the paracentral lobule in functional outcomes in the ARMS population. A restingstate functional MRI study in healthy subjects revealed that the paracentral lobule is functionally connected to other frontal and parietal regions, subserving motor functioning and spatial attention [63]. Of note, previous studies on schizophrenia demonstrated that some components of motor abnormalities (e.g., gesture deficits and neurological soft signs) and attention impairment [64], which can be caused by paracentral deficits [65] and be associated with social deficits [66, 67], predicted poor 1year functional outcomes [68]. Although the present study suggested an altered CT of the paracentral lobule to be a potential predictor of functional decline, further studies of more detailed motor-related abnormalities and disturbed attention subdomain are required to assess whether it can predict functional outcomes in high-risk cohorts.

In the ARMS individuals who underwent BACS, we found that $C T$ reduction in the superior and middle temporal gyrus, and that in the precentral gyrus and superior frontal gyrus are associated with poor motor speed and impaired verbal fluency, respectively. The results were partly similar to the associations between gray matter volume and cognitive functioning in schizophrenia patients $[69,70]$ and ARMS-P individuals [71, 72], supporting the existence of cognitive deficits even before psychosis onset as a trait vulnerability marker [73]. Baseline cognition, particularly in verbal fluency and verbal memory in ARMS individuals, was also reported to predict short- (i.e., 1-year follow-up) [74, 75] and medium to long-term ( 3-8 years) [64, 75, 76] functional outcomes. Although we failed to detect a significant relationship between the baseline BACS score and functional outcome in a subsample of our ARMS cohort $(n=63)$, future studies with a larger cohort should further examine the potential contribution of baseline cognition and its neuronal underpinnings to prediction of the clinical course of high-risk individuals.

This study has several limitations. First, although we processed all MR images similarly and included the scanning sites as covariates in the statistical model, the effects of scanner differences (Supplementary Table 1) may have confounded the findings. However, the current "applied" meta-analytic method and harmonization technique can better manage inter-scanner variances and largely replicated our main results (Supplementary Tables 6, 7 and Supplementary Figs. 2, 3). Second, we were unable to exclude potential confounding effects from antipsychotic 
medication. However, the antipsychotic medication dosage was not associated with CT in this ARMS cohort. As preceding studies reported altered $C T$ in medication-naive subjects of early psychosis $[77,78]$, our main findings may not be explained solely by antipsychotic medication. Lastly, a few previous studies have examined the relationship between brain structural/functional abnormalities and subsequent functional outcomes in ARMS individuals, and their definitions of functional outcome subgroups have been inconsistent $[34,79]$. While we used a novel definition to classify ARMS-R and -NR, the CT difference we found was replicated in the subanalyses using a more stringent definition that combined our definition with that in the previous study [34].

In conclusion, this multisite study demonstrated that ARMS individuals are characterized by cortical thinning in the frontotemporal regions and cortical thickening in the parieto-occipital regions, which may reflect general vulnerability to psychopathology. In addition, fronto-temporal cortical thinning may reflect neural underpinnings of cognitive impairments at the prodromal stage of psychosis. Moreover, cortical thinning of the right paracentral lobule in high-risk subjects may be associated with functional decline as a possible prognostic predictor.

\section{REFERENCES}

1. Melle I, Larsen TK, Haahr U, Friis S, Johannesen JO, Opjordsmoen S, et al. Prevention of negative symptom psychopathologies in first-episode schizophrenia: two-year effects of reducing the duration of untreated psychosis. Arch. Gen. Psychiatry. 2008;65:634-40.

2. Secher RG, Hjorthøj CR, Austin SF, Thorup A, Jeppesen P, Mors O, et al. Ten-year follow-up of the OPUS specialized early intervention trial for patients with a first episode of psychosis. Schizophr. Bull. 2015;41:617-26.

3. Takahashi T, Suzuki M, Tanino R, Zhou SY, Hagino H, Niu L, et al. Volume reduction of the left planum temporale gray matter associated with long duration of untreated psychosis in schizophrenia: a preliminary report. Psychiatry Res. 2007;154:209-19.

4. Bartholomeusz CF, Cropley VL, Wannan C, Di Biase M, McGorry PD, Pantelis C. Structural neuroimaging across early-stage psychosis: Aberrations in neurobiological trajectories and implications for the staging model. Aust. N. Z. J. Psychiatry. 2017;51:455-76.

5. Dietsche B, Kircher T, Falkenberg I. Structural brain changes in schizophrenia at different stages of the illness: A selective review of longitudinal magnetic resonance imaging studies. Aust. N. Z. J. Psychiatry. 2017;51:500-8.

6. Palaniyappan L, Marques TR, Taylor H, Handley R, Mondelli V, Bonaccorso S, et al. Cortical folding defects as markers of poor treatment response in first-episode psychosis. JAMA Psychiatry. 2013;70:1031-40.

7. Takahashi T, Takayanagi Y, Nishikawa Y, Nakamura M, Komori Y, Furuichi A, et al. Brain neurodevelopmental markers related to the deficit subtype of schizophrenia. Psychiatry Res Neuroimaging. 2017;266:10-18.

8. Takayanagi $Y$, Sasabayashi D, Takahashi T, Komori Y, Furuichi A, Kido M, et al. Altered brain gyrification in deficit and non-deficit schizophrenia. Psychol. Med. 2019;49:573-80.

9. Sasabayashi D, Takayanagi $Y$, Takahashi T, Nemoto K, Furuichi A, Kido M, et al. Increased brain gyrification in the schizophrenia spectrum. Psychiatry Clin. Neurosci. 2020a;74:70-76.

10. Yung AR, Yuen HP, McGorry PD, Phillips LJ, Kelly D, Dell'Olio M, et al. Mapping the onset of psychosis: the Comprehensive Assessment of At-Risk Mental States. Aust. N. Z. J. Psychiatry. 2005;39:964-71.

11. Seiler N, Nguyen T, Yung A, O'Donoghue B. Terminology and assessment tools of psychosis: A systematic narrative review. Psychiatry Clin. Neurosci. 2020;74:226-46.

12. Nelson B, Yuen HP, Wood SJ, Lin A, Spiliotacopoulos D, Bruxner A, et al. Longterm follow-up of a group at ultra high risk ("prodromal") for psychosis. The PACE 400 Study. JAMA Psychiatry. 2013;70:793-802.

13. Carrión RE, McLaughlin D, Goldberg TE, Auther AM, Olsen RH, Olvet DM, et al. Prediction of functional outcome in individuals at clinical high risk for psychosis. JAMA Psychiatry. 2013;70:1133-42.

14. Addington J, Cornblatt BA, Cadenhead KS, Cannon TD, McGlashan TH, Perkins $\mathrm{DO}$, et al. At clinical high risk for psychosis: outcome for nonconverters. Am. J. Psychiatry. 2011;168:800-5.

15. Fusar-Poli P, Nelson B, Valmaggia L, Yung AR, McGuire PK. Comorbid depressive and anxiety disorders in 509 individuals with an at-risk mental state: impact on psychopathology and transition to psychosis. Schizophr. Bull. 2014;40:120-31.
16. Takahashi T, Higuchi Y, Komori Y, Nishiyama S, Nakamura M, Sasabayashi D, et al Quality of life in individuals with attenuated psychotic symptoms: Possible role of anxiety, depressive symptoms, and socio-cognitive impairments. Psychiatry Res. 2017;257:431-7.

17. Fusar-Poli $P$, Bonoldi I, Yung AR, Borgwardt S, Kempton MJ, Valmaggia L, et al. Predicting Psychosis: Meta-analysis of transition outcomes in individuals at high clinical risk. JAMA Psychiatry. 2012;69:220-9.

18. Reniers RL, Lin A, Yung AR, Koutsouleris N, Nelson B, Cropley VL, et al. Neuroanatomical predictors of functional outcome in individuals at ultra-high risk for psychosis. Schiozophr Bull. 2017;43:449-58.

19. Lin A, Wood SJ, Nelson B, Beavan A, McGorry P, Yung AR, et al. Outcomes of nontransitioned cases in a sample at ultra-high risk for psychosis. Am. J. Psychiatry. 2015;172:249-58.

20. Hutton C, Draganski B, Ashburner J, Weiskopf N. A comparison between voxelbased cortical thickness and voxel-based morphometry in normal aging. Neuroimage. 2009;48:371-80.

21. Pereira JB, Ibarretxe-Bilbao N, Marti MJ, Compta $Y$, Junqué $C$, Bargallo $N$, et al. Assessment of cortical degeneration in patients with Parkinson's disease by voxel-based morphometry, cortical folding, and cortical thickness. Hum. Brain Mapp. 2012;33:2521-34

22. van Erp TGM, Walton E, Hibar DP, Schmaal L, Jiang W, Glahn DC, et al. Cortical Brain Abnormalities in 4474 Individuals With Schizophrenia and 5098 Control Subjects via the Enhancing Neuro Imaging Genetics Through Meta Analysis (ENIGMA) Consortium. Biol. Psychiatry. 2018;84:644-54.

23. Schultz CC, Koch K, Wagner G, Roebel M, Schachtzabel C, Gaser C, et al. Reduced cortical thickness in first episode schizophrenia. Schizophr. Res. 2010;116:204-9.

24. Crespo-Facorro B, Roiz-Santiáñez R, Pérez-Iglesias R, Rodriguez-Sanchez JM, Mata I, Tordesillas-Gutierrez $\mathrm{D}$, et al. Global and regional cortical thinning in firstepisode psychosis patients: relationships with clinical and cognitive features. Psychol. Med. 2011;41:1449-60.

25. van Haren NE, Schnack HG, Cahn W, van den Heuvel MP, Lepage C, Collins L, et al. Changes in cortical thickness during the course of illness in schizophrenia. Arch. Gen. Psychiatry. 2011;68:871-80.

26. Cobia DJ, Csernansky JG, Wang L. Cortical thickness in neuropsychologically nearnormal schizophrenia. Schizophr. Res. 2011;133:68-76.

27. Oertel-Knöchel V, Knöchel C, Rotarska-Jagiela A, Reinke B, Prvulovic D, Haenschel $C$, et al. Association between psychotic symptoms and cortical thickness reduction across the schizophrenia spectrum. Cereb. Cortex. 2013;23:61-70.

28. Yan J, Cui Y, Li Q, Tian L, Liu B, Jiang T, et al. Cortical thinning and flattening in schizophrenia and their unaffected parents. Neuropsychiatr. Dis. Treat 2019;15:935-46.

29. Behdinan T, Foussias G, Wheeler AL, Stefanik L, Felsky D, Remington G, et al Neuroimaging predictors of functional outcomes in schizophrenia at baseline and 6-month follow-up. Schizophr. Res. 2015;169:69-75.

30. Rodriguez-Perez N, Ayesa-Arriola R, Ortiz-García de la Foz V, Setien-Suero E, Tordesillas-Gutierrez D, Crespo-Facorro B, et al. Long term cortical thickness changes after a first episode of non- affective psychosis: The 10 year follow-up of the PAFIP cohort. Prog. Neuropsychopharmacol. Biol. Psychiatry. 2020;108:110180.

31. Jung WH, Kim JS, Jang JH, Choi JS, Jung MH, Park JY, et al. Cortical thickness reduction in individuals at ultra-high-risk for psychosis. Schizophr. Bull. 2011;37:839-49.

32. Tognin S, Riecher-Rössler A, Meisenzahl EM, Wood SJ, Hutton C, Borgwardt SJ, et al. Reduced parahippocampal cortical thickness in subjects at ultra-high risk for psychosis. Psychol. Med. 2014;44:489-98.

33. Klauser P, Zhou J, Lim JK, Poh JS, Zheng H, Tng HY, et al. Lack of Evidence for Regional Brain Volume or Cortical Thickness Abnormalities in Youths at Clinical High Risk for Psychosis: Findings From the Longitudinal Youth at Risk Study. Schizophr. Bull. 2015;41:1285-93.

34. de Wit S, Wierenga LM, Oranje B, Ziermans TB, Schothorst PF, van Engeland $H$, et al. Brain development in adolescents at ultra-high risk for psychosis: Longitudinal changes related to resilience. Neuroimage Clin. 2016;12:542-9.

35. Mizuno M, Suzuki M, Matsumoto K, Murakami M, Takeshi K, Miyakoshi T, et al. Clinical practice and research activities for early psychiatric intervention at Japanese leading centres. Early Inter. Psychiatry. 2009;3:5-9.

36. Koike $S$, Takano $Y$, Iwashiro N, Satomura $Y$, Suga $M$, Nagai T, et al. A multimodal approach to investigate biomarkers for psychosis in a clinical setting: the integrative neuroimaging studies in schizophrenia targeting for early intervention and prevention (IN-STEP) project. Schizophr. Res. 2013;143:116-24.

37. Inada T, Inagaki A. Psychotropic dose equivalence in Japan. Psychiatry Clin. Neurosci. 2015;69:440-7.

38. Miller TJ, McGlashan TH, Rosen JL, Cadenhead K, Cannon T, Ventura J, et al. Prodromal assessment with the structured interview for prodromal syndromes and the scale of prodromal symptoms: predictive validity, interrater reliability, and training to reliability. Schizophr. Bull. 2003;29:703-15. 
39. Hall RC. Global assessment of functioning. A modified scale. Psychosomatics. 1995;36:267-75.

40. American Psychiatric Association. Diagnostic and Statistical Manual of Mental Disorders. 4th ed. Washington, DC: American Psychiatric Press; 1994.

41. International Early Psychosis Association Writing Group. International clinical practice guidelines for early psychosis. Br J Psychiatry Suppl 48, s120-124 (2005).

42. Kaneda $Y$, Sumiyoshi T, Keefe R, Ishimoto $Y$, Numata $S$, Ohmori T, et al. Brief assessment of cognition in schizophrenia: validation of the Japanese version. Psychiatry Clin. Neurosci. 2007;61:602-9.

43. Sasabayashi D, Takayanagi $Y$, Takahashi $T$, Katagiri N, Sakuma A, Obara C, et al. Subcortical Brain Volume Abnormalities in Individuals With an At-risk Mental State. Schizophr. Bull. 2020b;46:834-45.

44. Fischl B. FreeSurfer. Neuroimage. 2012;62:774-81.

45. Hagler DJ Jr., Saygin AP, Sereno MI. Smoothing and cluster thresholding for cortical surface-based group analysis of fMRI data. Neuroimage. 2006;33:1093-103.

46. van Erp TG, Hibar DP, Rasmussen JM, Glahn DC, Pearlson GD, Andreassen OA, et al. Subcortical brain volume abnormalities in 2028 individuals with schizophrenia and 2540 healthy controls via the ENIGMA consortium. Mol. Psychiatry. 2016;21:547-53.

47. Okada N, Fukunaga M, Yamashita F, Koshiyama D, Yamamori H, Ohi K, et al. Abnormal asymmetries in subcortical brain volume in schizophrenia. Mol. Psychiatry. 2016;21:1460-6.

48. Johnson WE, Li C, Rabinovic A. Adjusting batch effects in microarray expression data using empirical Bayes methods. Biostatistics. 2007;8:118-27.

49. Fortin JP, Cullen N, Sheline YI, Taylor WD, Aselcioglu I, Cook PA, et al. Harmonization of cortical thickness measurements across scanners and sites. Neuroimage. 2018;167:104-20.

50. Benetti S, Pettersson-Yeo W, Hutton C, Catani M, Williams SC, Allen P, et al. Elucidating neuroanatomical alterations in the at risk mental state and first episode psychosis: a combined voxel-based morphometry and voxel-based cortical thickness study. Schizophr. Res. 2013;150:505-11.

51. Cannon TD, Chung Y, He G, Sun D, Jacobson A, van Erp TG, et al. Progressive reduction in cortical thickness as psychosis develops: a multisite longitudinal neuroimaging study of youth at elevated clinical risk. Biol. Psychiatry. 2015;77:147-57.

52. Fornito A, Yung AR, Wood SJ, Phillips LJ, Nelson B, Cotton S, et al. Anatomic abnormalities of the anterior cingulate cortex before psychosis onset: an MRI study of ultra-high-risk individuals. Biol. Psychiatry. 2008;64:758-65.

53. Takayanagi Y, Kulason S, Sasabayashi D, Takahashi T, Katagiri N, Sakuma A, et al. Reduced Thickness of the Anterior Cingulate Cortex in Individuals With an At-Risk Mental State Who Later Develop Psychosis. Schizophr. Bull. 2017;43:907-13.

54. Del Re EC, Stone WS, Bouix S, Seitz J, Zeng V, Guliano A, et al. Baseline cortical thickness reduction in clinical high risk for psychosis: Brain regions associated with conversion to psychosis versus non-conversion as assessed at one-year follow-up in the Shanghai-At-Risk-for-Psychosis (SHARP) study. Schizophr. Bull. 2021;47:562-74.

55. Jalbrzikowski M, Hayes RA, Wood SJ, Nordholm D, Zhou JH, Fusar-Poli P, et al. Association of structural magnetic resonance imaging measures with psychosis onset in individuals at clinical high risk for developing psychosis An ENIGMA working group mega-analysis. JAMA Psychiatry. 2021;78:753-66.

56. Takayanagi Y, Kulason S, Sasabayashi D, Takahashi T, Katagiri N, Sakuma A, et al. Structural MRI Study of the Planum Temporale in Individuals With an At-Risk Mental State Using Labeled Cortical Distance Mapping. Front Psychiatry. 2020;11:593952.

57. Lebedev AV, Beyer MK, Fritze F, Westman E, Ballard C, Aarsland D, et al. Cortical changes associated with depression and antidepressant use in Alzheimer and Lewy body dementia: an MRI surface-based morphometric study. Am. J. Geriatr. Psychiatry. 2014;22:4-13.e1.

58. Nesvåg R, Lawyer G, Varnäs $K$, Fjell AM, Walhovd KB, Frigessi $A$, et al. Regional thinning of the cerebral cortex in schizophrenia: effects of diagnosis, age and antipsychotic medication. Schizophr. Res. 2008;98:16-28.

59. Dukart J, Smieskova R, Harrisberger F, Lenz C, Schmidt A, Walter A, et al. Agerelated brain structural alterations as an intermediate phenotype of psychosis. J. Psychiatry Neurosci. 2017;42:307-19.

60. Kurachi M. Pathogenesis of schizophrenia: Part I. Symptomatology, cognitive characteristics and brain morphology. Psychiatry Clin. Neurosci. 2003a;57:3-8.

61. Kurachi M. Pathogenesis of schizophrenia: Part II. Temporo-frontal two-step hypothesis. Psychiatry Clin. Neurosci. 2003b;57:9-15.

62. Thompson PM, Sowell ER, Gogtay N, Giedd JN, Vidal CN, Hayashi KM, et al. Structural MRI and brain development. Int Rev. Neurobiol. 2005;67:285-323.

63. Salvador R, Suckling J, Coleman MR, Pickard JD, Menon D, Bullmore E, et al. Neurophysiological architecture of functional magnestic resonance images of human brain. Cereb. Cortex. 2005;15:1332-42.
64. Sawada K, Kanehara A, Sakakibara E, Eguchi S, Tada M, Satomura Y, et al. Identifying neurocognitive markers for outcome prediction of global functioning in individuals with first-episode and ultra-high-risk for psychosis. Psychiatry Clin. Neurosci. 2017;71:318-27.

65. Hirjak D, Wolf RC, Stieltjes B, Hauser T, Seidl U, Schröder J, et al. Cortical signature of neurological soft signs in recent onset schizophrenia. Brain Topogr. 2014;27:296-306.

66. Walther S, Stegmayer K, Sulzbacher J, Vanbellingen T, Müri R, Strik W, et al. Nonverbal social communication and gesture control in schizophrenia. Schizophr. Bull. 2015;41:338-45.

67. Prouteau A, Helene V, Briand C, Lesage A, Lalonde P, Nicole L, et al. Cognitive predictors of psychosocial functioning outcome in schizophrenia: A follow-up study of subjects participating in a rehabilitation program. Schizophr. Res. 2005;77:343-53.

68. Prikryl R, Ceskova E, Kasparek T, Kucerova H. Neurological soft signs and their relationship to 1-year outcome in first-episode schizophrenia. Eur. Psychiatry. 2007;22:499-504.

69. Baaré WF, Hulshoff Pol HE, Hijman R, Mali WP, Viergever MA, Kahn RS. Volumetric analysis of frontal lobe regions in schizophrenia: relation to cognitive function and symptomatology. Biol. Psychiatry. 1999;45:1597-605.

70. Suazo V, Díez Á, Montes C, Molina V. Structural correlates of cognitive deficit and elevated gamma noise power in schizophrenia. Psychiatry Clin. Neurosci. 2014;68:206-15.

71. Hannan KL, Wood SJ, Yung AR, Velakoulis D, Phillips LJ, Soulsby B, et al. Caudate nucleus volume in individuals at ultra-high risk of psychosis: a cross-sectional magnetic resonance imaging study. Psychiatry Res. 2010;182:223-30.

72. Meijer JH, Schmitz N, Nieman DH, Becker HE, van Amelsvoort TA, Dingemans PM, et al. Semantic fluency deficits and reduced grey matter before transition to psychosis: a voxelwise correlational analysis. Psychiatry Res. 2011;194:1-6.

73. Fusar-Poli P, Deste G, Smieskova R, Barlati S, Yung AR, Howes O, et al. Cognitive functioning in prodromal psychosis: a meta-analysis. Arch. Gen. Psychiatry. 2012;69:562-71.

74. Meyer EC, Carrión RE, Cornblatt BA, Addington J, Cadenhead KS, Cannon TD, et al. The relationship of neurocognition and negative symptoms to social and role functioning over time in individuals at clinical high risk in the first phase of the North American Prodrome Longitudinal Study. Schizophr. Bull. 2014;40:1452-61.

75. Bolt LK, Amminger GP, Farhall J, McGorry PD, Nelson B, Markulev C, et al. Neurocognition as a predictor of transition to psychotic disorder and functional outcomes in ultra-high risk participants: Findings from the NEURAPRO randomized clinical trial. Schizophr. Res. 2019;206:67-74.

76. Lin A, Wood SJ, Nelson B, Brewer WJ, Spiliotacopoulos D, Bruxner A, et al. Neurocognitive predictors of functional outcome two to 13 years after identification as ultra-high risk for psychosis. Schizophr. Res. 2011;132:1-7.

77. Narr KL, Toga AW, Szeszko P, Thompson PM, Woods RP, Robinson D, et al. Cortical thinning in cingulate and occipital cortices in first episode schizophrenia. Biol. Psychiatry. 2005;58:32-40.

78. Rais M, Cahn W, Schnack HG, Hulshoff Pol HE, Kahn RS, van Haren NE, et al. Brain volume reductions in medication-naive patients with schizophrenia in relation to intelligence quotient. Psychol. Med. 2012;42:1847-56.

79. van Haren NE, Hulshoff Pol HE, Schnack HG, Cahn W, Brans R, Carati I, et al. Progressive brain volume loss in schizophrenia over the course of the illness: Evidence of maturational abnormalities in early adulthood. Biol. Psychiatry. 2008;63:106-13.

\section{ACKNOWLEDGEMENTS}

This study was supported by JSPS KAKENHI Grant Numbers JP18K15509 to DS, JP26461738 and JP18K07549 to YT, JP18K07550 to T, JP19H03579 to SK, JP20KK0193 to DS and SK, JP24390281 and JP2OH03598 to MSuz, JP16H06395, JP16H06399, JP16K21720, JP16H06280, and JP2OH03596 to KK, the SENSHIN Medical Research Foundation to YT and DS, THE HOKURIKU BANK Grant-in-Aid for Young Scientists to DS, and by the Health and Labour Sciences Research Grants for Comprehensive Research on Persons with Disabilities from the Japan Agency for Medical Research and Development (AMED) Grant Number JP16dk0307029h0003 to MSuz, KM, and MM. The study was also supported in part by AMED under Grant Numbers JP20dm0307001, JP20dm0307004, and JP20dm0207069, Moonshot R\&D Grant Number JPMJMS2021, UTokyo Center for Integrative Science of Human Behavior (CiSHuB), and by World Premier International Research Center Initiative (WPI), MEXT, Japan to KK.

\section{COMPETING INTERESTS}

The authors declare no competing interests. 


\section{ADDITIONAL INFORMATION}

Supplementary information The online version contains supplementary material available at https://doi.org/10.1038/s41398-021-01516-2.

Correspondence and requests for materials should be addressed to D.S.

Reprints and permission information is available at http://www.nature.com/ reprints

Publisher's note Springer Nature remains neutral with regard to jurisdictional claims in published maps and institutional affiliations.
Open Access This article is licensed under a Creative Commons Attribution 4.0 International License, which permits use, sharing, adaptation, distribution and reproduction in any medium or format, as long as you give appropriate credit to the original author(s) and the source, provide a link to the Creative Commons license, and indicate if changes were made. The images or other third party material in this article are included in the article's Creative Commons license, unless indicated otherwise in a credit line to the material. If material is not included in the article's Creative Commons license and your intended use is not permitted by statutory regulation or exceeds the permitted use, you will need to obtain permission directly from the copyright holder. To view a copy of this license, visit http://creativecommons. org/licenses/by/4.0/.

(c) The Author(s) 2021 